\title{
INTEGRATION OF THE DISCRETE SINE-GORDON EQUATION WITH A SELF-CONSISTENT SOURCE
} @ B.A. Babajanov, A.K. Babadjanova, A.Sh. Azamatov a.murod@mail.ru,oygul@bk.ru,azizbek.shavkatovich@gmail.com

\section{УДК 517.518}

DOI: $10.33184 /$ mnkuomsh2t-2021-10-06.9.

This work is devoted to the application of the inverse scattering theory for integration of the discrete Sine-Gordon equation with a self-consistent source.

Keywords: discrete sine-Gordon equation, self-consistent source, discrete Dirac-type operator, scattering data, inverse scattering method

Hirota showed the integrabilty of discrete version of the sine-Gordon equation and found its Lax pair, Backlund transformations and N-soliton solutions [1]. In [2] generalization of Hirota's discretization scheme for the sine-Gordon equation was considered. The soliton solutions are obtained by extending the generalized inverse method [3] and the related linear spectral problem for the discrete sine-Gordon equation was studied in [4].

It needs to point out the $\mathrm{sG}$ equations and its close allies are valued in the investigation of a great variety of diverse fields[5], such as the study of surfaces with constant negative curvature, or integrable surfaces [6], elementary particle physics, quantum optics, Josephson junctions [7], nonlinear excitations in condensed matter physics[8], vortex structures in fluids and plasmas [9].

This study investigated the integration of the discrete sine-Gordon(sG) equation with a self-consistent source via the inverse scattering method.

The first investigation of the soliton equations with self-consistent sources has been considered in [10] and still attracts considerable attention in recent years [11-13, see also their reference].

We consider the following system of equations

$$
\begin{gathered}
\dot{\theta}_{n+1}-\dot{\theta}_{n}=2\left(\sin \theta_{n+1}+\sin \theta_{n}\right)+\sum_{k=1}^{N}\left(f_{1, n+1}^{k} f_{1, n}^{k}+f_{2, n+1}^{k} f_{2, n}^{k}\right), n \in Z, \\
\left.\theta_{n}(t)\right|_{t=0}=\theta_{n}^{0}, n \in Z,
\end{gathered}
$$

Babajanov Bazar Atajanovich, DSc, Dotsent, Urgench State University, (Urgench, Uzbekistan);

Babadjanova Oygul Komiljanovna, PhD, Urgench State University, (Urgench, Uzbekistan);

Azamatov Azizbek SHavkatovich, PhD student, Urgench State University, (Urgench, Uzbekistan); 


$$
L_{n}\left(z_{k}, t\right) f_{n}^{k}=f_{n+1}^{k}, n \in Z
$$

where $L_{n}(z, t)=z P_{n}(t)+\frac{1}{z} Q_{n}(t), P_{n}(t)=\frac{1}{2}\left(\begin{array}{cc}1+\cos \theta_{n}(t) & \sin \theta_{n}(t) \\ \sin \theta_{n}(t) & 1-\cos \theta_{n}(t)\end{array}\right)$,

$$
Q_{n}(t)=\frac{1}{2}\left(\begin{array}{cc}
1-\cos \theta_{n}(t) & -\sin \theta_{n}(t) \\
-\sin \theta_{n}(t) & 1+\cos \theta_{n}(t)
\end{array}\right),
$$

$\theta_{n}=\theta_{n}(t)$ and $f_{n}^{k}=f_{n}\left(z_{k}, t\right), \hat{f}_{n}^{k}=\sigma_{2} f_{n}\left(z_{k}, t\right)$ column-vector functions satisfy the following normalizing conditions

$$
\beta_{k}(t)=\sum_{i=-\infty}^{\infty}\left(f_{i}^{k}\right)^{T}\left(Q_{i}-P_{i}\right) \sigma_{2} f_{i}^{k}, \quad \hat{\beta}_{k}(t)=\sum_{i=-\infty}^{\infty}\left(\hat{f}_{i}^{k}\right)^{T}\left(z_{k}^{2} Q_{i}-P_{i}\right) \sigma_{2} \hat{f}_{i}^{k} .
$$

Here $\beta_{k}(t), \hat{\beta}_{k}(t)$ are given scalar continuous functions and $\sigma_{i}(i=1,2,3)$ are the usual Pauli matrices of rank 2.

The purpose of the work is to find the set of functions $\left\{\theta_{n}(t), f_{n}^{k}(t)\right\}, n \in$ $Z$ supposing the existence in the class of functions

$$
\lim _{|n| \rightarrow \pm \infty} \omega_{n}=\lim _{|n| \rightarrow \pm \infty} \sum_{j=n}^{\infty} \sin \theta_{j}(t)=0, \quad \begin{gathered}
\theta_{n}(t)=0(\bmod 2 \pi) \\
|n| \rightarrow \infty
\end{gathered}
$$

which is the solution of the considering (1)-(3) problem.

Theorem 1. If the set of functions $\left\{\theta_{n}(t), f_{n}^{k}(t)\right\}$ represent the solution of the (1)-(3) in the class of functions (4), then the scattering data of the $L_{n}(t)$ operator with the potential $\theta_{n}(t)$ satisfy the following time evolution equations

$$
\begin{gathered}
\dot{R}(z)=-2\left(\frac{z^{2}+1}{z^{2}-1}\right) R(z), \quad|z|=1, \quad z \neq \pm 1, \\
\dot{z}_{k}=0, \dot{C}_{k}=\left(-2 \frac{z_{k}^{2}+1}{z_{k}^{2}-1}+\beta_{k}+\hat{\beta}_{k}\right) C_{k}, k=1, \ldots, N .
\end{gathered}
$$

The obtained results completely define the time evolution of the scattering data, which allows us to solve the problem (1)-(3) by using the method of the inverse spectral problem of (3) [see 4].

\section{Литература}

1. Hirota R. 1977, J. Phys. Soc. Japan, 43, 2079.

2. Orfanidis S. 1978 J. Phys. Rev. D 183822.

3. Levi D., Ragnisco O., Bruschi M. 1980, Nuovo Cimento A 5856.

4. Pilloni L. and Levi $D$. The Inverse Scattering Transform for Solving the Discrete Sine-Gordon Equation // Physics Letters A, 92 (1982), 1, pp. 5-81982. 
5. Fritz Gesztesy, Helge Holden. A Local Sine-Gordon Hierarchy and its AlgebroGeometric Solutions // arXiv:solv-int/9707010

6. Bobenko A. I. Constant mean curvature surfaces and integrable systems // Russ. Math. Surv.46:4 , 1991, 1-45.

7. Borisov A.B., Kiseliev V.V. Topological defects in incommensurate magnetic and crystal structures and quasi-periodic solutions of the elliptic sine-Gordon equation // Physica D $31,1988,49-64$.

8. Ting A.C., Chen H.H., Lee Y.C. Exact solutions of a nonlinear boundary value problem: The vortices of the two-dimensional sinh-Poisson equation // Physica 26D , 1987, pp.37-66.

9. Mel'nikov V.K. Integration method of the Korteweg-de Vries equation with a self-consistent source // Phys.Lett. A, 133, 1988, pp.493-496.

10. Da-Jun Zhang, Deng-yuan Chen. The N-Soliton Solutions of the sineGordon Equation with Self-Consistent Sources // Physica A 321, 2003, pp. 467-481.

11. Khasanov A.B., Urazboev G.U. On the sine-Gordon equation with a selfconsistent source // Mat. Tr., 2008, Volume 11, Number 1, 153-166,

12. Babajanov B.A., Fechkan M., Urazbaev G.U. On the periodic Toda Lattice with self-consistent source // Communications in Nonlinear Science and Numerical Simulation. 2015; 22: 379-388. 